\title{
Editorial
}

\section{Why Are Antibiotic-Resistant Nosocomial Infections Spiraling Out of Control?}

\author{
Carlene A. Muto, MD, MS
}

For as long as the Centers for Disease Control and Prevention (CDC) has measured the prevalence of hospital-acquired infections caused by multidrug-resistant organisms, it has been increasing. ${ }^{1-4}$ According to the CDC, more than $70 \%$ of the bacteria now causing hospitalacquired infections are resistant to at least one of the drugs most commonly used to treat them. ${ }^{5}$ It seems important to control these infections because they have been more costly and more deadly than those due to antibiotic-susceptible strains of the same species.-9 Nevertheless, despite decades of discussing the control of antibiotic-resistant nosocomial infections, there has been little evidence of control in most healthcare facilities in most countries. ${ }^{1-3}$ Several articles in this issue of Infection Control and Hospital Epidemiology evaluate the efforts that have been made by hospitals to control them and may help to explain this failure. ${ }^{10-14}$

The first of these, by L'Hériteau et al., ${ }^{10}$ reports the findings of a nationwide, prospective study designed to describe methods used for diagnosis and surveillance of nosocomial infections and multidrug-resistant organisms in French intensive care units (ICUs). They sought to survey all 573 French ICUs, but only $252(44 \%)$ responded to the written survey. Follow-up telephone interviews of personnel in $143(47 \%)$ of the initially nonresponding ICUs allowed them to increase the overall response rate to $69 \%$. Despite the existence of clearly stated French national guidelines, ${ }^{15}$ the authors found that there were "profound differences" in the way that ICUs had implemented the recommendations for surveying for various nosocomial infections. They concluded that great caution must be exercised when compar- ing data regarding ICU infections among French hospitals. Regarding surveillance for multidrug-resistant organisms, they found that $70 \%$ of the respondents performed active surveillance cultures to detect these organisms on admission, whereas $60 \%$ did so during the stay; $88 \%$ of the responding ICUs flagged carriers of multidrug-resistant organisms so that isolation could be maintained consistently. This indicates important variation among hospitals, and inconsistent use of an effective control measure could help to explain difficulties in controlling multidrug-resistant organisms (ie, approximately one-third of the time active surveillance cultures were not being performed to identify contagious patients).

A second national survey in this issue of Infection Control and Hospital Epidemiology was designed to examine the extent of implementation of strategies to control multidrug-resistant organisms in U.S. hospitals based on recommendations from a 1994 workshop co-sponsored by the National Foundation for Infectious Diseases and the CDC.$^{16}$ Ward et al. ${ }^{11}$ surveyed hospitals for compliance with five goals to optimize antibiotic use and five goals to detect, report, and prevent spread of multidrug-resistant organisms. From approximately 7,000 U.S. hospitals, 108 hospitals $(1.5 \%)$ were randomly selected to receive a survey (excluding Veterans' Affairs hospitals and small- to medium-sized teaching hospitals). The survey response rate was $60 \%$. Approximately half of the responding hospitals were classified as large ( $>200$ beds) and an equal proportion of those remaining as small (50 to 99 beds) and medium (100 to 199 beds). Survey responses were recorded using a 5-point Likert scale. The mean response regarding

Dr. Muto is from the Division of Hospital Epidemiology and Infection Control, University of Pittsburgh Medical Center, Presbyterian Campus, Infectious Diseases Epidemiology Research Unit, University of Pittsburgh School of Medicine and Graduate School of Public Health, Pittsburgh, Pennsylvania. mutoca@upmc.edu

Address reprint requests to Carlene A. Muto, MD, MS, University of Pittsburgh Medical Center (UPMC-P), 1215 Kaufmann Building, Pittsburgh, PA 15213. 
each strategy was less than 4 , indicating only moderate compliance. Nevertheless, they found no differences regarding bed size, teaching status, or geographic region, suggesting that hospitals of all sizes and types may have potential to successfully implement such goals. The authors concluded that hospitals need to do more to improve antimicrobial use and to detect, report, and control the spread of multidrug-resistant organisms. The use of active surveillance cultures to detect multidrug-resistant organisms to ensure appropriate isolation, a major focus of the French national study ${ }^{10}$ and also of a recent Society for Healthcare Epidemiology of America (SHEA) guideline for preventing the transmission of multidrug-resistant strains of Staphylococcus aureus and Enterococcus, ${ }^{17}$ was not mentioned in this study of U.S. hospitals, perhaps because the National Foundation for Infectious Diseases-CDC workshop recommendations on which it was based did not comment on this approach.

Flach et al. ${ }^{12}$ conducted a sophisticated study designed to assess U.S. hospitals' methods for identifying bacterial antimicrobial resistance, reporting it to clinicians, and monitoring its spread in a timely manner. Five variables were studied: (1) disseminating antibiograms to physicians at least annually, (2) notifying physicians of antimicrobial-resistant infections, (3) reporting susceptibility results within 24 hours, (4) using automated testing procedures, and (5) offering molecular typing. Eighty-five (78\%) of 106 U.S. hospitals selected to receive the survey responded. The use of individual procedures ranged from $33 \%$ to $85 \%$. The authors concluded that this represented great variability and that hospitals are not doing enough to monitor spread. Once again, active surveillance cultures were not mentioned despite the fact that recent studies have suggested that $85 \%$ of all hospitalized patients colonized with methicillin-resistant $S$. aureus (MRSA) or vancomycin-resistant Enterococcus (VRE) go unrecognized and unisolated in the absence of active surveillance cultures ${ }^{18,19}$ and that patients with multidrug-resistant organisms cared for using standard precautions have been significantly more likely to transmit them than have those cared for using contact precautions. ${ }^{20,21}$

The article by Lee et a1. ${ }^{13}$ in this issue of Infection Control and Hospital Epidemiology examines the sensitivity and cost-effectiveness associated with three active surveillance culture strategies for identifying VRE-colonized patients. At the study onset, routine active surveillance cultures for VRE were being performed for patients in highrisk areas (ie, hematology-oncology unit, bone marrow and solid organ transplant unit, and surgical and medical ICUs). This strategy was referred to as CURRENT and was compared with two additional strategies: screening the CURRENT patients plus those with a history of renal disease (RENAL) and screening CURRENT patients plus those hospitalized in the previous 2 years (HOSP). For each of the surveillance strategies, the costs of screening and of hospitalization per admission were calculated and the survival rate was estimated.

In addition to their CURRENT strategy, 200 consecu- tive stool specimens submitted for Clostridium difficile testing were screened for VRE. Seventeen of the 81 specimens submitted from high-risk units were positive for VRE; 16 of these were also identified via CURRENT methods. The expanded testing identified 5 non-high-risk VRE colonizations. All 22 patients identified as colonized with VRE had been hospitalized within the prior 2 years. Only 3 of the 5 non-high-risk patients had a history of renal impairment. VRE cultures of diarrheal stool specimens submitted for $C$. difficile screening would have resulted in missing $71 \%$ of the patients colonized with VRE. The HOSP strategy would involve screening the greatest number of patients (all those with a hospital admission in the prior 2 years), resulting in slightly higher screening costs; however, this strategy resulted in greater recognition of currently undetected colonization and was associated with lower overall costs due to improved control of VRE and optimal estimated survival rates. For these reasons, the authors advocated expanding VRE surveillance programs to include patients hospitalized in the prior 2 years.

As mentioned above, Ward et al. concluded that efforts to control the inappropriate use of antimicrobials were insufficient in many U.S. hospitals. In this issue of Infection Control and Hospital Epidemiology, Bolon et al. ${ }^{14}$ report frequent inappropriate use of vancomycin in the pediatric wards of a single hospital almost a decade after the $\mathrm{CDC}$ made recommendations regarding how vancomycin use should be controlled. ${ }^{22}$ Fortunately, a metaanalysis from the same university found that vancomycin use was not a particularly important risk factor for VRE acquisition, ${ }^{23}$ but this does not mean that it cannot play some role in potentiating the survival and spread of VRE. Inappropriate use is also associated with excess costs and the risk of unnecessary side effects for patients. This study serves to reinforce the conclusion of Ward et al. that despite decades of trying, we have not yet become very good at optimizing antimicrobial use.

As stated earlier, there has been widespread failure to control antibiotic-resistant nosocomial infections due to pathogens such as MRSA and VRE. The five studies discussed in this editorial suggest that a major reason for this failure of control has been the frequent failure of hospitals to use effective measures. What should be done to rectify this situation? Hospitals should begin following an evidence-based approach. The combination of strategies advocated by the SHEA guideline on control of MRSA and VRE, ${ }^{17}$ emphasizing identification and containment of spread, is the most effective method documented to date for controlling multidrug-resistant organisms. Notably, it has been recommended by the $\mathrm{CDC}$ for control of "important" pathogens ranging from severe acute respiratory syndrome to vancomycin-resistant $S$. aureus. ${ }^{24,25}$ The recent, draft Healthcare Infection Control Practices Advisory Committee guideline proposed intensifying prevention interventions for multidrug-resistant organisms whenever there is evidence of their continuing transmission within a facility. ${ }^{26}$ As the overall prevalence of multidrug-resistant organisms continues to increase nationally, ${ }^{2,4}$ one might 
suspect that there is evidence of continuing transmission in most, if not all, U.S. healthcare facilities, which have primarily relied on standard precautions as most patients colonized with multidrug-resistant organisms have not been sought, identified, or isolated. By contrast, hospitals that have used the approach advocated in the SHEA guideline have repeatedly shown control of endemic or epidemic MRSA and VRE infections. Twenty-five such reports were presented at the past two SHEA annual meetings.

\section{REFERENCES}

1. Centers for Disease Control and Prevention. National Nosocomial Infections Surveillance (NNIS) System report: data summary from January 1990-May 1999. Am J Infect Control 1999;27:520-532.

2. Centers for Disease Control and Prevention. NNIS Antimicrobial Resistance Report: Vancomycin-Resistant Enterococcus (VRE) Facts. Atlanta, GA: Centers for Disease Control and Prevention; 1999. Available at www.cdc.gov/phtn/old/vrefacts.htm.

3. Centers for Disease Control and Prevention. National Nosocomial Infection Surveillance (NNIS) System report: data summary from January 1992-June 2001. Am J Infect Control 2001;29:404-421.

4. Centers for Disease Control and Prevention. Proportion of HealthcareAcquired S. aureus Infections Resistant to Oxacillin (MRSA) Among ICU Patients, 1989-2003. Atlanta, GA: Centers for Disease Control and Prevention; 2004. Available at www.cdc.gov/ncidod/hip/ARESIST/ mrsa.htm.

5. Centers for Disease Control and Prevention. Campaign to Prevent Antimicrobial Resistance in Healthcare Settings: Why a Campaign? Atlanta, GA: Centers for Disease Control and Prevention; 2001. Available at www.cdc.gov/drugresistance/healthcare/problem.htm.

6. Cosgrove SE, Sakoulas G, Perencevich EN, Schwaber MJ, Karchmer AW, Carmeli Y. Comparison of mortality associated with methicillinresistant and methicillin-susceptible Staphylococcus aureus bacteremia: a meta-analysis. Clin Infect Dis 2003:36:53-59.

7. Cosgrove SE, Qi Y, Kaye KS, Harbarth S, Karchmer AW. The impact of methicillin resistance in Staphylococcus aureus bacteremia on patient outcomes: mortality, length of stay and hospital charge. Presented at the 41st Annual Meeting of the Interscience Conference on Antimicrobial Agents and Chemotherapy; September 22-25, 2001; Chicago, IL. Abstract K-1221:415.

8. Engemann JJ, Carmeli Y, Cosgrove SE, et al. Adverse clinical and economic outcomes attributable to methicillin resistance among patients with Staphylococcus aureus surgical site infection. Clin Infect Dis 2003;36:592-598.

9. Salgado CD, Farr BM. Outcomes associated with vancomycin resistant enterococci: a meta-analysis. Infect Control Hosp Epidemiol 2003;24:690698.

10. L'Hériteau F, Alberti $C$, Cohen $Y$, Troché G, Moine P, Timsit J-F. Nosocomial infection and multidrug-resistant bacteria surveillance in intensive care units: a survey in France. Infect Control Hosp Epidemiol 2005;26:13-20

11. Ward MM, Diekema DJ, Yankey JW, et al. Implementation of strategies to prevent and control the emergence and spread of antimicrobial-resistant microorganisms in U.S. hospitals. Infect Control Hosp Epidemiol 2005;26:21-30.

12. Flach SD, Diekema DJ, Yankey JW, et al. Variation in the use of procedures to monitor antimicrobial resistance in U.S. hospitals. Infect Control Hosp Epidemiol 2005;26:31-38.

13. Lee TA, Hacek DM, Stroupe KT, Collins SM, Peterson LR. Three surveillance strategies for vancomycin-resistant enterococci in hospitalized patients: detection of colonization efficiency and a cost-effectiveness model. Infect Control Hosp Epidemiol 2005;26:39-46.

14. Bolon $\mathrm{MK}$, Arnold $\mathrm{AD}$, Feldman $\mathrm{HA}$, et al. Evaluating vancomycin use at a pediatric hospital: new approaches and insights. Infect Control Hosp Epidemiol 2005;26:47-55.

15. Comité Technique National des Infections Nosocomiales. 100 Recommendations for Surveillance and Prevention of Nosocomial Infections. Paris: Ministere de l'Emploi et de la Solidarité; 1999.

16. Goldmann DA, Weinstein RA, Wenzel RP, et al. Strategies to prevent and control the emergence and spread of antimicrobial-resistant microorganisms in hospitals. JAMA 1996;275:234-240.

17. Muto CA, Jernigan JA, Ostrowsky BE, et al. SHEA guideline for preventing nosocomial transmission of multidrug-resistant strains of Staphylococcus aureus and Enterococcus. Infect Control Hosp Epidemiol 2003;24:362-386.

18. Calfee DP, Giannetta ET, Durbin LJ, Farr BM. Control of endemic vancomycin-resistant Enterococcus (VRE) among inpatients at a university hospital. Clin Infect Dis 2003;37:326-332.

19. Salgado CD, Farr BM. What proportion of hospital patients colonized with methicillin-resistant Staphylococcus aureus are identified by clinical microbiology cultures? Presented at the 13th Annual Meeting of the Society for Healthcare Epidemiology of America; April 5-8, 2003; Arlington, VA.

20. Vriens MR, Fluit AC. Troelstra A, Verhoef J, Van Der Werken C. Are MRSA more contagious than MSSA in a surgical intensive care unit? Infect Control Hosp Epidemiol 2002;23:491-494.

21. Jernigan JA Titus MG, Groschel DHM, Getchell-White SI, Farr BM. Effectiveness of contact isolation during a hospital outbreak of methicillin-resistant Staphylococcus aureus. Am I Epidemiol 1996;143:496504.

22. HICPAC. Recommendations for preventing the spread of vancomycin resistance: Hospital Infection Control Practices Advisory Committee (HICPAC). Infect Control Hosp Epidemiol 1995;16:105-113.

23. Carmeli Y, Samore MH, Huskins C. The association between antecedent vancomycin treatment and hospital-acquired vancomycin-resistant enterococci: a meta-analysis. Arch Intern Med 1999;159:2461-2468.

24. Centers for Disease Control and Prevention. Public Health Guidance for Community-Level Preparedness and Response to SARS. Atlanta, GA: Centers for Disease Control and Prevention; 2004. Available at www.cdc.gov/ncidod/sars/guidance/index.htm.

25. Centers for Disease Control and Prevention. Health Department and Infection Control Guidance: Investigation and Control of Vancomycin Intermediate and Resistant Staphylococcus aureus (VISA/VRSA). Atlanta, GA: Centers for Disease Control and Prevention; 2004. Available at www.cdc.gov/ncidod/hip/ARESIST/visa_vrsa_guide.pdf.

26. Centers for Disease Control and Prevention. Draft guideline for isolation precautions: preventing transmission of infectious agents in healthcare settings, 2004. Federal Register 2004;69:33034. 